\title{
Les essais thérapeutiques en néonatologie
}

L'évaluation des médicaments chez le nouveau-né est nécessaire en raison des particularités pharmacodynamiques et pharmacocinétiques propres à cet âge de la vie. Le retard à l'évaluation des médicaments dans cette tranche d'âge est expliqué par les difficultés de réalisation des essais, l'application de la loi de protection des personnes et le faible marché représenté par la prescription en néonatologie. Les règles méthodologiques classiques s'appliquent sans restriction à la néonatologie avec certaines particularités dues aux rapides modifications physiologiques de l'organisme en développement. La quantification des effets des médicaments, souvent difficile en néonatologie, nécessite l'adaptation des outils de mesure de ces effets.

\section{Elisabeth Autret Annie-Pierre Jonville}

\section{ADRESSE}

E. Autret : professeur des universités, praticien hospitalier. A.-P. Jonville : assistante hospitalouniversitaire. Service de pharmacologic clinique, hôpital Bretonneau, 2 bis, boulcvard 'évaluation des médicaments nécessitc la réalisation d'essais thérapeutiques selon une méthodologie maintenant établie. Nous cnvisagcrons successivement la situation actuelle de ces essais en néonatologie, leur justification et leur méthologie.

\section{Situation actuelle des essais en néonatologie}

Le retard relatif à l'évaluation correcte des médicaments en néonatologie et le manque d'expérience des néonatologistes dans le domaine des essais tiennent en grande partie à l'étroitesse du marché, au caractère facultatif de ces essais, et aux difficultés d'application de la loi portant sur la protection des personnes qui se prêtent aux recherches biomédicales [1-6].

L'étroitesse du marché

On comprend le peu de motivation des firmes pharmaceutiques à investir dans l'évaluation d'un médicament destiné à une tranche d'âge où les retombées financières seront légè- res, si on les compare à celles de sectcurs clés tels que celui de la cardiologie ou des médicaments du vieillissement. Cela est d'autant plus vrai que la plupart des médicaments concernés possèdent déjà une autorisation de mise sur le marché (AMM) délivrée pour d'autres indications, notamment chez l'adulte, et sont souvent prescrits par extrapolation au nouveau-né, des données recueillies chez l'adulte. Quel industriel consentirait, par exemple, à financer la mise en place d'un essai destiné à évaluer l'effet des gluco-corticoïdes dans la bronchodysplasie séquellaire d'une maladie des membranes hyalines?

Le caractère facultatif de cette recherche

Les autorités d'enregistrement n'imposent qu'exceptionnellement des essais en néonatologie avant d'accorder une AMM. C'est ainsi que les antibiotiques prescrits très fréquemment sont encore trop souvent commercialisés sans étude spécifique en néonatologie, et que des psychotropes puissants comme le midazolam et le fentanil [7, 8] ont été utilisés pour la sédation des nouveau-nés, avant que 
leur pharmacocinétique, leur pharmacodynamie et encore moins leur intolérance ne soient évaluées. Ces mêmes autorités pourraient, d'une part, inciter les firmes à mettre en place des études, dès l'obtention d'une certitude de sécurité et d'efficacité laissant présumer une activité intéressante en néonatologie, et, d'autre part, avertir les prescripteurs de la contre-indication des médicaments non évalués.

\section{La législation}

La loi portant sur la protection des personnes qui se prêtent aux recherches biomédicales votée le 20 décembre 1988 est d'application difficile en néonatologie $[3,9,10]$.

\section{- La défense des droits des enfants}

La défense des droits de l'individu souhaitée par cette loi est assurée par le comité consultatif de protection des personnes. Ce comité donne son avis sur la pertinence du projet, l'adéquation entre son objectif et les moyens mis en œuvre pour sa réalisation, ainsi que sur la qualification des investigateurs. Il analyse tout particulièrement la protection et les termes du consentement demandé au sujet. Dans la majorité des cas, ces comités de protection ne comportent pas de pédiatres et encore moins de néonatologistes. Il paraît cependant indispensable qu'un expert en néonatologie soit consulté afin de juger de la pertinence scientifique du projet, avant émission de l'avis éthique.

\section{- Son champ d'application}

Cette loi concerne toute étude visant à développer la connaissance médicale ou biomédicale. Mais ces recherches ne peuvent se faire que si elles sont fondées sur le dernier état des connaissances, sur une expérience préclinique suffisante et si leur risque prévisible n'est pas disproportionné avec le bénéfice attendu. Ces essais sont effectués sous la direction d'un pédiatre justifiant d'une expérience appropriée et dans des conditions matérielles et techniques adaptées à l'essai et compatibles avec les impératifs de rigueur scientifique et de sécurité des personnes.

La recherche en pédiatrie peut être effectuée à condition qu'on en attende un bénéficc direct pour la santé des enfants. Ce préambule est difficile à satisfaire, car l'appréhension du béné$\mathrm{m} / \mathrm{s} n^{\circ} 3$ vol. 9, mars 93 fice est parfois délicate s'il s'agit d'un médicament strictement destiné au nouveau-né (surfactant).

Les recherches sans bénéfice individuel direct, représentées essentiellement par les études pharmacocinétiques, sont admises en néonatologie, à certaines conditions. Elles doivent être utiles à des personnes présentant les mêmes caractéristiques d'âge, de maladie ou de handicap. Cette recommandation est facile à suivre parce que les données pharmacocinétiques sont nécessaires, et que celles obtenues chez un enfant ne lui seront pas directement utiles mais le seront à d'autres. Si ces études sont éthiquement possibles avec un médicament potentiellement utile à l'enfant, il serait en revanche inacceptable de donner un médicament dans le seul but d'obtenir des données pharmacocinétiques. Les prélèvements répétés nécessaires à ces études, peu contraignants en présence d'un cathéter, sont douloureux s'ils sont obtenus par ponction. Ils s'accompagnent toujours d'une spoliation sanguine. Ces études doivent ne pas pouvoir être réalisées autrement, ce qui est souvent le cas puisqu'aucun modèle expérimental n'est utilisable en néonatologie. Les dosages sur de petites quantités de sang et les études simplifiées utilisant un petit nombre de prélèvements contribuent à respecter ces recommandations. Si ces études simplifiées ne fournissent pas de données aussi précises que les études classiques, les renseignements qu'elles permettent d'obtenir sont souvent suffisants. L'absence de risque sérieux prévisiblc exigée par la loi est beaucoup plus ambiguë en raison de la difficulté d'appréciation du risque. En effet, cette appréciation est nécessairement biaisée par l'intérêt que l'investigateur voit à cette étude. La réflexion au cas par cas par un groupe d'experts serait à conseiller.

\section{- Le consentement}

L'une des plus sérieuses difficultés auxquelles sont confrontés les néonatologistes est probablement celle du consentement. La loi exige un consentement libre, éclairé et écrit. Pour que le consentement soit libre, il faut que les sujets soient indépendants de l'investigateur et de l'expérimentateur. Pour que le consentement soit éclairé, le sujet doit être averti des conditions de réalisation de l'étude, des bénéfices attendus, des contraintes, des risques prévisibles, de l'avis du comité de protection des personnes et de son droit de refuser ou de retirer à tout moment son consentement. Pour que le consentement soit réel, il doit être donné par écrit ou, en cas d'impossibilité, attesté par un tiers. De nombreux médecins acceptent mal l'idée du consentement écrit, qui modifie la relation médecinmalade. Cependant, il ne dégage en rien le médecin de sa responsabilité et doit être compris comme un droit à l'information, allant dans le sens d'un plus grand respect des malades. Le consentement en néonatologie, donné par les parents ou le tuteur légal et qui se rapproche d'une autorisation, est indiscutablement difficile à obtenir. D'une part, parce que la présence simultanée des deux parents est exceptionnelle et, d'autre part, parce qu'il est plus simple de s'engager que d'engager autrui, surtout s'il s'agit de son propre enfant. Les parents sont partagés entre le désir de savoir et de décider, et celui de laisser au médecin la responsabilité de la décision. Les parents craignent une mauvaise tolérance s'ils donnent l'autorisation, et de priver leur enfant d'un éventuel traitement utile s'ils la refusent. Le problème est singulièrement plus compliqué lorsqu'il s'agit d'unc situation d'urgence comme celle d'une naissance difficile. Un séjour en réanimation et la séparation immédiate de la mère et de son enfant rend cette clause inacceptable et vécue comme une agression supplémentaire [11]. L'aide à la décision par un groupe constitué de néonatologistes, de parents et de personnel infirmier serait une solution non prévue par la loi.

Le consentement est encore plus difficile à demander et à obtenir s'il s'agit d'un essai sans bénéfice individuel direct.

\section{Justification de l'évaluation des médicaments en néonatologie}

\section{La nécessité d'essais thérapeutiques en néonatologie}

L'évaluation des médicaments en néonatologie est nécessaire, en raison 


\section{RÉFÉRENCES}

1. Autret E. Essais thćrapeutiques des médicaments en pédiatric. Arch Fr Pediatr 1988 ; $45: 155-6$.

2. Autret E. Modalités générales de mise en place des essais des médicaments en pédiatric. Arch Fr Pediatr 1990; 47 : 627-8.

3. Autret E. Implications éthiques et pratiques en pédiatric de la loi portant sur la protection des personnes se prêtant aux recherches biomédicales. Arch Fr Pediatr $1991 ; 48: 457-9$

4. Autret E, Jonville AP. La prescription médicamenteuse chez le nouveau-né et la responsabilité médicale. In : Soutoul JH, Laugier J, eds. La Responsabilité médicale et les problèmes médico-légaux en néonatologie. Paris : Maloine, 1991 : 164-74.

5. Grodin MA, Alpert JJ. Children as participants in medical research. Pediatr Clin North Am 1988 ; 35 : 1389-401.

6. Janofsky J, Starficld B. Assessment of risk in research on children. J Pediatr 1981 ; 98 : 842-5.

7. Arnold JH, Truog RD, Scavone JM, Fenton $T$. Changes in the pharmacodynamic response to fentanyl in neonates during continuous infusion. J Pediatr 1991; 119 : 639-43

8. Lane JC, Tennison M, Lawless ST, Greenwood RS, Zaritsky AL. Movement disorder after withdrawal or fentanyl infusion. J Pediatr 1991; 119 : 649-51.

9. Chabas F. I a loi sur les essais thérapcutiques. Médecine génćrale. Rev Prat 1989 ; $72: 115-24$

10. Loi $\mathrm{n}^{\circ} 88-1138$ du 20 décembre 1988 modifiće, relative à la protection des personnes qui se prêtent à des recherches biomédicales. Journal Officiel de la République Française du 22 décembre $1988 ; 16032-5$ du 25 janvier $1990 ; 1009-14$ du 29 septembre 1990

11. Dawson J. Randomised trials and informed consent in neonatal medicine. $\mathrm{Br} \mathrm{Med}$ J $1986 ; 292$ : 1373-4.

12. Morselli P. Influence du développement sur la pharmacocinćtique chez le nouveauné, le nourrisson et l'enfant. Arch Fr Pediatr $1986 ; 33: 151-6$

13. W. Kendind J, Rubens LJ, Notter RH, Davis JN, Cox Ch. A comparison of surfactant as immediate prophylaxis and as rescue therapy in newborns of less than 30 wecks' gestation. N Engl J Med 1991 ; $324: 865-71$

14. Avery ME, Allen Merritt T. Surfactantreplacement therapy. $N$ Engl J Med 1991 ; 324: 910-2.

15. Eschwege E, Bouvenot G, Doyon F, Lacroux A. Essais thérapeutiques: mode d'emploi. Paris : Éditions Inserm, 1990.

16. Bryce RL, Enkin MW. Six months about controlled trials in perinatal medecine. Am J Obstet Gynecol 1985 ; 151 : 707-10.

17. Brazclton TB. Neonatal Behavioral assessment scale. London: William Heinemann Medical Books, 1973.

18. Owen ME. Pain in infancy : conceptual and methodological issues. Pain 1984;20 : 213-30.

19. McGrath PJ, Cunningham SJ, Goodman JT, Unruh A. The clinical measurement of pain in children : a review. Clin $J$ des particularités cinétiques [12] et dynamiques décrites dans la tranche d'âge concernée. Ces particularités rendent impossible l'extrapolation $a$ priori au nouveau-né des données obtenues chez l'adulte ou chez l'enfant plus grand.

L'élimination hépatique et rénale est ralentie pendant la période néonatale et se traduit par une prolongation de la demi-vie. Un espacement des prises ou une réduction des doses de médicaments dont l'index thérapeutique est étroit évite la toxicité due à un surdosage.

Les particularités pharmacodynamiques sont moins bien appréhendées bien que fréquemment évoquées à propos des effets indésirables particuliers à l'organisme en développement. Citons le grey baby syndrome dû au chroramphénicol et le retentissement sur la croissance, prouvé dans le cas des rétinoïdes et suspecté dans celui des quinolones. Enfin, les thérapeutiques des désordres exclusivement pédiatriques incluant toute la néonatologie ne peuvent ĉtre évaluées que dans cette tranche d'âge. Les raisons éthiques aux essais des médicaments en néonatologie sont claires, si l'on admet qu'il n'est pas éthique de priver un enfant d'un médicament potentiellement actif par contreindication systématique ou de l'utiliser sans évaluation pédiatrique.

\section{Les modalités de mise en place des essais}

Une réflexion commune aux modalités de mise en place des essais par des pédiatres, des pharmacologues cliniciens et des industriels est sûrement une façon pragmatique de progresser dans l'évaluation des médicaments en néonatologie [2]. Le schéma du développement en néonatologie a pour objectif de présenter aux autorités d'enregistrement un dossier complet. Les moyens de l'obtenir exigent de persuader les investigateurs de cette nécessité, et de convaincre les firmes pharmaceutiques que l'investissement présente un intérêt sinon financier du moins de prestige.

Les études qui s'adressent à des volontaires sains (phase 1) ne concernent pas l'enfant.

Les études de pharmacocinétique sont entreprises en pédiatrie après l'obtention de données chez l'adulte, et en utilisant une forme galénique adaptée aux enfants. La dose de départ est calculée $a$ priori à partir des posologies adultes, en tenant compte des modifications pharmacocinétiques habituellement observées chez les nouveau-nés. En raison de la maturation rapide des systèmes d'élimination rénale et hépatique en fonction de l'âge gestationnel et postnatal, ces évaluations sont effectuées chez les prématurés et chez les nouveau-nés à terme âgés de 1 à 7 jours et de 7 jours à 1 mois. Il s'agit le plus souvent d'études réalisées après administration d'une dose unique à un petit effectif de nouveau-nés. La mesure des concentrations plasmatiques après des doses répétées n'est indispensable que lorsque le caractère prolongé du traitement fait craindre une accumulation dont les conséquences cliniques peuvent être délétères. Les études de pharmacocinétique en néonatologie nécessitent des adaptations techniques, telles que les dosages sur de très petites quantités de sang, déjà évoqués. La faible sécrétion salivaire du nouveau-né rend peu réalistes les dosages salivaires. La pharmacocinétique dite de population, dont le principe est de réduire le nombre de prélèvements en augmentant l'effectif étudié, est certainement une méthode d'avenir pour l'évaluation pharmacocinétique en néonatologie.

Tous les médicaments destinés à être prescrits à des nouveau-nés doivent être préalablement évalués dans cette tranche d'âge. Dans les affections strictement identiques chez le nouveau-né et chez l'adulte - qui, à notre connaissance, n'existent quasiment pas - seules des études de tolérance seraient envisagées en néonatologie, mais celles-ci ne permettent aucune conclusion en l'absence de comparaison à un traitement de référence ou à un placebo. La condition préalable à ces études est la connaissance de la pharmacologie animale, identique à ce que l'on exige chez l'adulte, complétée par l'étude des conséquences sur le développement cérébral et osseux. Le moment le plus opportun pour ces études d'efficacité (phase 3 ) en néonatologie dépend du type d'affection et du degré d'innovation attendu du médicament. S'il s'agit d'une maladie banale dotée d'une alternative thérapeutique et 
d'un produit peu innovant, ou si l'affection cst intermédiaire et/ou cxclusivement néonatologique [13, 14], l'étude de phase 3 scra cntreprisc chez l'enfant, après avoir acquis des renscignements suffisants sur le rapport bénéfice/risque chez l'adultc (phase 4). Ce sont les situations les plus fréquentes, notamment celles des antibiotiques et des médicaments du reflux gastro-œsophagien. En revanche, si la maladie est grave, sans alternative thérapeutique (SIDA, canccr) et si le produit apparaît innovant, la phase 3 pédiatrique sera menée parallèlement à la phase 3 adultc. L'absence d'essai de phase 3 pédiatrique doit être claircment motivée par une contrc-indication chez l'enfant.

\section{La méthodologie des essais des médicaments}

Fondée sur des principes fondamentaux bien détcrminés [15, 16], ellc est applicable à la néonatologic avec les contraintes éthiques déjà envisagécs et certaines particularités tcchniques.

\section{La comparaison}

L'intérêt d'un médicament ne peut ĉtre évalué dans l'absolu, mais uniquement par comparaison à un autrc traitement. Cette comparaison est bien souvent anecdotique, personnelle, et nc pcut donc prétendre convaincre une assemblée scientifique. Pour avoir une valeur généralc, la comparaison doit porter sur un cnscmble de malades répartis en deux groupes, l'un recevant lc traitement à évaluer, l'autrc ne le recevant pas, appclé groupc témoin. Cc groupe témoin peut ne pas être traité, recevoir un placebo ou cncorc un médicament de référence utilisé, bien sûr, dans ses conditions optimales d'emploi. Ainsi, l'évaluation d'un nouvel antibiotique en néonatologie doit le comparer au meillcur antibiotique disponible, utilisé dans le schéma posologique proposé dans cette tranche d'âge. Il cst alors rare d'observer unc différence d'efficacité, il s'agira tout au plus d'unc absence de différence assortie d'une meillcure tolérance ou d'une pharmacocinétique plus intéressante.

Il est licite de comparcr le médica$\mathrm{m} / \mathrm{s} n^{\circ} 3$ vol. 9, mars 93 ment à évaluer à un placebo s'il n'existe pas de traitement connu de la maladie, si la maladic est bénigne ou spontanément curable. Lc surfactant a été ainsi comparé à un placebo, en l'absence de médicament de référence. Au manque d'enthousiasme des néonatologistes à utiliser le placebo, on pcut opposer, outre les arguments méthodologiques déjà cnvisagés, qu'il n'y a aucunc raison de penser que l'effet placebo est absent chez lc nouveau-né et que peu de médicaments sont suffisamment évalués pour servir de médicament de référence. De plus, la différence avec lc traitement à évaluer, plus grandc avec un placcbo qu'avec un médicament de référencc, scra plus rapidc à mettre en évidence, ce qui permettra de proposer plus vite un médicament intéressant.

\section{La causalité}

La causalité est le sccond point fort méthodologique. La différence entre dcux groupes traités différemment nc peut êtrc attribuéc au traitcment que si ces deux groupes (traité et témoin) sont strictement comparables (âge, facteurs pronostiques, traitements associés), excepté par la naturc des traitements comparés et cc, à l'inclusion et tout au long de l'essai.

Lc scul moyen d'assurer que les groupes sont comparables au début dc l'étude est d'attribuer lc traitement par tirage au sort. En effet, si l'on maîtrise l'attribution des traitements, la tentation est forte d'inclure lc malade qui paraît plus grave dans le groupc traitement à évalucr plutôt que dans le groupc témoin. Le tirage au sort est l'une des procédures qui confèrent aux essais lcur valeur scientifique, et comme sa mise en œuvre concerne des patients pour lesquels on est en droit d'hésiter au sujet du mcilleur traitement possible, il n'y a pas de contrc-indication éthique à son utilisation.

La seulc procédurc permettant d'assurer la comparaison des groupes à la fin de l'essai cst le double aveugle. Comment, en effet, évaluer de la même façon au cours de toute une étude, des patients dont on sait que l'un reçoit le traitement supposé actif et l'autre lc placebo ou le médicament de référence? La connaissance par le médecin du traitement reçu, alors que le patient ou son entourage l'ignore (technique de simplc aveugle), induit dans l'évaluation des résultats les mêmes biais que ccux des essais ouverts. L'évaluation par un médecin censé ignorer le traitement reçu est également illusoire à l'intérieur d'une même équipc. Ainsi tout devra être mis en œuvre pour travailler en double aveugle, y compris lorsque les voies d'administration ou les formes galéniques sont différentes, situations au cours desquelles il est possible d'utiliser un double placcbo.

\section{Le degré de signification}

Lc résultat d'un cssai s'évalue par la présence ou l'absence d'unc différence significativc. La différence est dite significative au risque de $5 \%$ $(p<0,05)$ s'il y a moins de 5 chances sur 100 pour que la différence mise en évidence soit duc au hasard. Ce risque de mettre en évidence unc différence non due au traitement (risque $\alpha$ ) mais due simplement au hasard (fluctuations d'échantillon) cst admis à $5 \%$. En revanche, le risque d'absence de mise en évidence d'unc différence qui cxiste en réalité (risque $\beta$ ), calculé à la fin dc l'essai, pcut révéler un manque de puissance, le plus souvent en rapport avec un effectif insuffisant.

Lc nombre de sujets nécessaires est calculé avant le début de l'étude et son calcul claircment justifié témoignc que l'essai cnvisagé a fait l'objet d'unc réflexion suffisante. Ce nombre constitue un ordre de grandeur, scul moyen pour le clinicien de juger si l'cssai cst réalistc. Un effectif nécessaire trop important par rapport au recrutement des investigateurs, conduit à renoncer à l'essai ou à proposcr une étude pluricentrique.

Le nombre de sujets dépend de quatre paramc̀tres : de la taille de la différence cliniquement intércssante, fixée à l'avance par le clinicien, que l'on souhaite mettre en évidence entre les traitements comparés (plus cette différence est grande, moins il faudra de patients) ; de la variabilité du critère de jugement (qui nécessite un nombre de patients d'autant plus élevé qu'elle cst importante) ; du risque $\alpha$, accepté à $5 \%$; ct de la puissance du test de comparaison qui est la probabilité (fixée en général à 80 
ou à $90 \%$, soit un risque $\beta$ de $10 \%$ à $20 \%$ ) d'cstimer une différence entre les effets de deux traitements si cette différence existe vraiment.

\section{La question à laquelle veut répon-} dre l'essai

L'étude a pour objectif principal de répondre à une seule question principale et à la bonne. Celle-ci doit ĉtre clairement définic et formuléc à l'avance. Ainsi, chercher à démontrer que les glucocorticoïdes sont efficaces pour traiter la broncho-dysplasie n'a pas de sens. En effet, veut-on savoir s'ils diminuent la durée de la ventilation assistée ou la fréquencc de ses séquelles pulmonaires? Ce ne sont ni les mêmes malades ni les mêmes critères dc jugement qui apporteront ces réponses. Des objectifs dits secondaires peuvent ĉtre intéressants (sécurité d'cmploi, interactions médicamenteuses, acceptabilité), mais ils alourdissent l'essai et compromettent ses chances d'aboutir. Un effort de réflexion préalable sélectionne les questions associécs les plus pertinentes.

Définir la question à laquelle un essai thérapeutique vcut répondre revient donc à définir un critc̀re de jugement principal. Sans vouloir négliger l'appréciation globalc du médecin, force est d'admettre que la comparaison de deux traitements passe nécessairement par le choix raisonné et toujours discutablc du meilleur critère possible pour mesurer l'efficacité. Ce critère doit ĉtre unique car c'est lui qui servira au calcul du nombre de sujets. Il sera défini comme tel et sans ambiguïté dans lc protocole, tout comme les critc̀res secondaircs sur lesquels il aura toujours la priorité et dont il ne faut pas majorer l'importance au vu des résultats. Ce critère doit ĉtre pertinent, fiable, facile à mesurer, disponible pour tous les investigateurs. Quel est, par excmple, le bon critère de jugement de l'évaluation d'un antibiotique dans les colonisations bactériennes néonatales ? Le nombre de nouveau-nés chez lesqucls les germes pathogènes ont disparu, ou la fréquence de survenue d'une méningitc précoce? Le bon critère de jugement de l'évaluation du surfactant est-il le délai de sevrage de la ventilation assistée, ou la fréquence des survies sans séquelle?
Le critère de jugement est parfois facile à mesurer, comme par exemple la température ou la fraction inspiréc d'oxygènc, mais souvent l'outil de mesure [17-19] n'est pas validé en néonatologie (échelles d'hétéroévaluation de la douleur et de la sédation). Pour des raisons techniques et éthiques, les explorations non invasives (échographie, doppler) méritent d'ĉtre développées.

\section{La sélection des patients}

La sélection des patients est nécessaire, mĉme s'il existe une contradiction entre le désir de rigueur et le souci de représentativité, qui voudrait que l'on puisse extrapoler au plus grand nombre les résultats d'un essai ayant porté sur quelques dizaines ou, mieux, quelques centaincs de paticnts. Il faut cependant définir la maladie par des critères précis, simples, non ambigus, d'obtention rapide et aisée.

Si certains factcurs ont une valeur pronostique dans l'affection étudiée, et que l'on ne veut pas courir lc risque d'un déséquilibre dans la répartition de ce facteur entre les groupcs comparés, qui empêcherait d'attribucr au médicament une éventuelle différence, deux attitudes sont possibles : soit imposer comme critère d'inclusion la présence ou l'absence de cc critère pronostique, soit séparer les patients avant l'inclusion (stratification) entre ccux ayant et ceux n'ayant pas cc critc̀re, et effectuer un tirage au sort dans chacun de ces deux groupes. La plupart des affections néonatales ayant unc évolution spontanéc différentc en fonction de l'âge gestationnel, une stratification sur ce critc̀re cst souvent pertinente.

\section{Le plan expérimental}

Parmi les trois types d'essais, en groupes parallèles (l'un recevant lc traitement, l'autrc ne le recevant pas), séquenticl et en cross over (le sujet est son propre témoin et reçoit l'un puis l'autre traitement dans un ordre attribué par tirage au sort), ce dernier, bien que séduisant parce qu'il réduit lc nombre de sujets en diminuant la variabilité inter-sujets, est incompatible avec la situation physiologique évolutive qui caractérise la période néonatale.

\section{L'analyse}

Enfin, l'analyse des résultats doit ĉtre prévue et décrite à l'avance en précisant les variables qui seront analysées et les tests qui seront utilisés. Cela nécessite une discussion, avant le début de l'étude, entre le promoteur, les investigateurs et les méthodologistes.

\section{Conclusion}

Lcs essais thérapeutiques d'évaluation des médicaments en néonatologie sont indispensables en raison de la physiologie particulic̀re de la période néonatale. Lcur développement doit permettre d'administrer aux nouveau-nés des médicaments correctement évalués dont le rapport bénéficc/risquc soit positif. Ces essais requièrent l'implication de tous les partenaires, qu'il s'agisse des autorités d'enregistrement, des industriels et des néonatologistes. Toutes les règles méthodologiques s'appliquent sans restriction aux nouveau-nés, mais une adaptation des outils de mesure et des protocoles cxpérimentaux est indispensable

\section{Summary}

Drug trials in neonatal medicine

In view of the specific pharmacodynamics and pharmacokinetics in neonates, it is necessary to evaluate drugs for use in this age range. The difficulties of performing drug trials in neonates, the application of the law which protects individuals and the small market represented by neonatal prescription, all account for the delay in the evaluation of drugs for this group of patients. Classical methodological rules can be applied without exception to neonatology, but with particular attention to physiological development. The precise measurement of the effects of drugs, of ten difficult in neonatology, requires the adaptation of the means of measuring these effects.

\section{TIRÉS A PART}

E. Autret. 